\section{Immunmodulation mit oraler Darreichungsform und ihre Wirkmechanismen}

\author{
R. Gold ${ }^{1}$ \\ ${ }^{1}$ Neurologische Klinik, St. Josef Hospital, Ruhr Universität \\ Bochum
}

In diesem Jahr erwarten wir die Zulassung von bis zu drei neuen oralen Immuntherapien bei schubförmiger Multipler Sklerose (MS). Dazu zählen Teriflunomid (Aubagio, $14 \mathrm{mg} /$ Tag), Dimethylfumarat (BG12 - Tecfidera, $2 \times 240 \mathrm{mg} / \mathrm{Tag}$ ) und Laquinimod (Nerventra, 0,6 mg/Tag) Die erste Substanz hat im September die Zulassung erhalten, für die zweite Substanz hat das CHMP bereits eine positive Empfehlung gegeben, während Laquinimod sich noch im Prüfverfahren befindet.

Die neuen Medikamente können die Basistherapie der schubförmigen MS grundlegend verändern. Mit Dimethylfumarat kam eine Wirksubstanz in die MS-Therapie, wo viele glückliche Fügungen zusammenspielten. Am Anfang stand eine Reihe von Patienten mit MS und schwerer Psoriasis, die wegen der Hauterkrankungen mit einer Fumaratemischung der ersten Generation behandelt wurden. Zunächst nahm man an, dass die Fumarate ähnlich wie bei Psoriasis zu einer sogenannten Immunmodulation durch TH2 Mechanismen von T-Lymphozyten führen. Zusätzlich fanden wir, dass über ein Schlüsselprotein der antioxidativen Stoffwechselwege, das Nrf2, neuroprotektive Eigenschaften vermittelt werden. Es ist oft nicht einfach, unter experimentellen Bedingungen gewonnene Daten auf den Menschen zu übertragen, aber sowohl kernspintomografische Untersuchungen als auch Ergebnisse an Blutzellen sprechen dafür, dass solche Mechanismen auch beim Menschen aktiv wirken. Da das kleine Fumaratmolekül die Blut-Hirn-Schranke gut überwindet, kann es auch im entzündeten Nervensystem selbst seine Wirkung entfalten. Die beiden Phase-III-Studien konnten neben hoher Wirksamkeit auch ein sehr gutes Sicherheitsprofil des Medikamentes bestätigen.

Ähnlich zum Dimethylfumarat vermittelt auch Laquinimod sowohl immunologische als auch neuroprotektive Wirkungen. Ursprünglich wurde das Medikament aus dem Linomide entwickelt und deshalb die Dosis nur sehr vorsichtig gesteigert. Über mehrere Phase-II- und -III-Studien hat Laquinimod ein exzellentes Sicherheitsprofil gezeigt. Die hervorstechenden Ergebnisse der Phase-III-Zulassungsstudien waren die deutliche Verhinderung der Hirnatrophie durch die tägliche Gabe von 0,6 mg Laquinimod, verbunden mit einer Verzögerung der Krankheitsprogression. Umfangreiche experimentelle Untersuchungen belegen, dass die Inhibition von NFkappaB einen Schlüsselmechanismus darstellt, aber auch die Induktion von regulatorischen NK-Zellen. Zukünftig werden höhere Dosierungen untersucht werden, in der Erwartung dass dort auch stärkere entzündungshemmende Mechanismen vorkommen.

Teriflunomid übt einen sehr gut verträglichen, anhaltenden Effekt auf Lymphozyten aus, der unter anderem auch selektiv immunsuppressiv auf aktivierte Lymphozyten wirkt. Inwieweit noch zusätzlich immunomodulatorische Mechanismen aktiv sind, wird momentan intensiverforscht.

Interessenkonflikt: Ralf Gold erhielt Redner- und Consulting Honorare sowie wiss. Forschungsunterstützung von Biogen Idec, Bayer Schering, TEVA, Novartis, Merck Serono und Sanofi-Aventis.

\section{Bibliografie}

Dol http://dx.doi.org/10.1055/s-0033-1346709

Drug Res 2013; 63, Suppl. 1: S9-S9

(c) Georg Thieme Verlag KG Stuttgart · New York . ISSN 2194-9379 